\title{
Frequency Response Measurements Based Analysis of Small Signal Model of DC-DC Converter
}

\author{
Husan Ali1 ${ }^{*}$, Xiancheng Zheng1, Shahbaz Khan ${ }^{1}$, Haider Zaman ${ }^{1}$, Muhammad Saad ${ }^{2}$ \\ ${ }^{1}$ Department of Electrical Engineering, School of Automation, Northwestern Polytechnical University, Xi'an, \\ 710072, China. \\ 2 Department of Electronics and Control Engineering, Chang'an University, Xi'an, 710064, China. \\ * Corresponding author. Tel.: 008618740476 759; email: engr.husan@gmail.com \\ Manuscript submitted June 30, 2017; accepted September 30, 2017.
}

doi: 10.17706/ijcee.2018.10.2.72-84

\begin{abstract}
Two port network based modeling approach is used to obtain the analytical expressions for dc-dc buck converter. The analytical expressions obtained include the effect of parasitic elements. The parasitic resistance associated with the filter capacitor and inductor has a significant influence on the converter's frequency responses, so a very simple and systematic procedure has been introduced for the computation of series resistance associated with capacitor and inductor from measured frequency responses. Further, the obtained small signal model is validated through experiment. Once the analytical expressions are available, a prototype of the converter is implemented and frequency responses are measured for various parameters know as g-parameters both in open as well as closed loop mode. The experimental results are compared with those obtained using modeling and it is found that the measurement results match the analytical results. The frequency response measurement based validation of small signal model, presented for dc-dc buck converter, can be used for any type of dc-dc converter working in an open or closed loop configuration.
\end{abstract}

Key words: DC-DC converter, frequency response, g-parameters, parasitic elements, transfer function.

\section{Introduction}

Small signal models are used for the analysis and design of power converters used in a variety of applications [1]-[2]. In modern distributed power systems, several different converters are interfaced in various configurations [3]-[5]. Therefore, the accuracy of these models is significant for the analysis of a complete system. The accuracy of these models can be improved by including the effect of various parasitic elements. Though ignoring parasitic elements simplifies the modeling procedure, such models often fail to predict the exact behavior of the converter.

The conduction loss associated with elements, e.g. inductor and capacitor, is of primary significance and referred to as equivalent series resistance (ESR). The series resistance of output filter capacitor and inductor has a strong influence on the converter's dynamics. The ESR of a capacitor has a significant role in the controller design, so if any arbitrary values are assumed for parasitic elements, it may lead to an erroneous design of controller. In case of a non-ideal buck converter, the ESR of the capacitive filter introduces a zero to the frequency response. Therefore, a procedure has been developed to show the calculation of series resistance associated with capacitor and inductor from measured frequency response 
functions.

Two port network based modeling approach is used to model the dc-dc buck converter. The small signal models developed for dc-dc converters often lack experimental validation and the converters are assumed to be ideal. So in this work, after computing the analytical expressions, a prototype of buck converter is implemented and frequency response measurements are recorded for set of parameters know as g-parameters, which represent the internal dynamics of the converter. The frequency response measurements are done for both open and closed loop system. The results obtained from the experiment are compared with the simulation results done for analytical expressions. The close matching of the results validates the small signal model of the converter. The results are obtained for both open and closed loop systems. The procedure of frequency response measurements based validation of small signal model can be applied to other topologies of dc-dc converters as well, working in either open or closed loop configuration.

Fig. 1 shows the schematic circuit of the buck converter with voltage mode control. Different blocks such as power stage $G_{v d}$, output voltage sensor $H_{s e}$, feedback compensator $G_{c}$ and PWM generator $G_{m}$ are also highlighted.

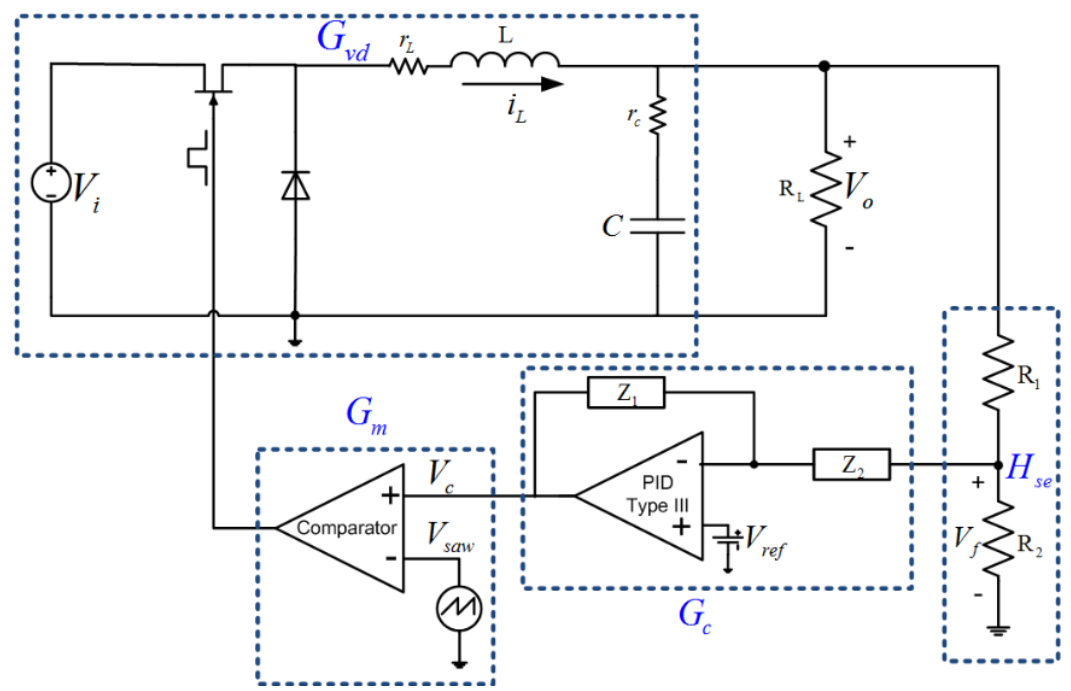

Fig. 1. DC-DC buck converter.

The rest of this paper is organized as follows. Section II presents the two port network based small signal modeling. Section III gives brief idea about the procedure used for frequency response measurements. Section IV compares the results obtained from simulation and experiment. Section V concludes of the paper.

\section{Two Port Model of Buck Converter}

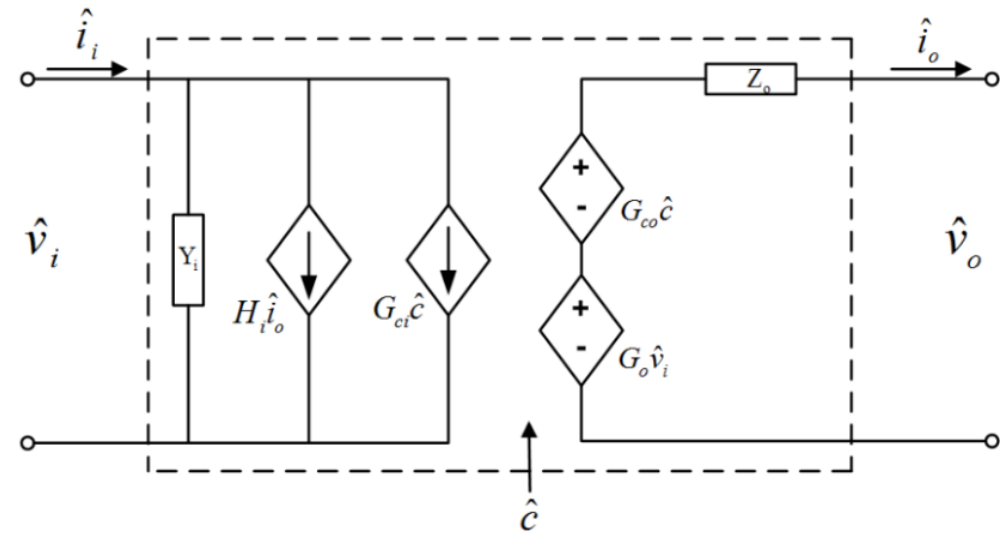

Fig. 2. Two port model of dc-dc converter with g-parameters. 
Two port models have been extensively applied to analyze DC-DC converters [6]-[8]. The use of linear two port representation based on g-parameters, represents the real internal dynamics excluding the source and the load effects. The input and output port parameters constitute of a set known as g-parameters. The set is composed of transfer functions which represent the characteristics of switched mode converter. The two port network representation of dc-dc converter is shown in Fig. 2 [9].

In matrix form the two port network shown in Fig. 2 can be represented by [9]

$$
\left[\begin{array}{l}
\hat{\imath}_{i} \\
\hat{v}_{o}
\end{array}\right]=\left[\begin{array}{ccc}
Y_{i-o} & H_{i-o} & G_{c i} \\
G_{o-o} & -Z_{o-o} & G_{c o}
\end{array}\right]\left[\begin{array}{c}
\hat{v}_{i} \\
\hat{\imath}_{o} \\
\hat{c}
\end{array}\right]
$$

The negative sign in output impedance is due to the chosen convention of the current flow $\hat{\mathrm{I}}_{\mathrm{o}}$. The subscript 'o' denotes that the parameters given are for open loop system. The closed loop g-parameters in terms of open loop parameters are given in (2).

$$
G(s)=\left[\begin{array}{cc}
Y_{i-c} & H_{i-c} \\
G_{o-c} & -Z_{o-c}
\end{array}\right]=\left[\begin{array}{cc}
Y_{i-o}-\frac{G_{o-o} G_{c i}}{G_{c o}} \frac{L(s)}{1+L(s)} & H_{i-o}+\frac{Z_{o-o} G_{c i}}{G_{c o}} \frac{L(s)}{1+L(s)} \\
\frac{G_{o-o}}{1+L(s)} & -\frac{Z_{o-o}}{1+L(s)}
\end{array}\right]
$$

Equation (2) makes it clear that the closed loop g-parameters can be calculated from the set of open loop g-parameters. After applying the basic circuit theory and state space averaging technique, the buck converter circuit in Fig. 1 can be represented as shown in (3)

$$
\begin{aligned}
& \frac{d \hat{\imath}_{L}}{d t}=-\frac{\left(r_{L}+D r_{S}+D^{\prime} r_{d}+r_{C}\right)}{L} \hat{\imath}_{L}-\frac{1}{L} \hat{v}_{c}+\frac{D}{L} \hat{v}_{i}+\frac{r_{C}}{L} \hat{\imath}_{o}+\frac{V_{i}+\left(r_{d}-r_{S}\right) I_{L}}{L} \hat{d} \\
& \frac{d \hat{v}_{C}}{d t}=\frac{\hat{i}_{L}}{C}-\frac{\hat{\imath}_{O}}{C} \\
& \hat{\imath}_{i}=D \hat{\imath}_{L}+I_{L} \hat{d} \\
& \hat{v}_{o}=\left(1+r_{c} C \frac{d}{d t}\right) \hat{v}_{c}
\end{aligned}
$$

In matrix form (3) can be written as

$$
\begin{aligned}
& {\left[\begin{array}{l}
\frac{d \hat{\imath}_{L}}{d t} \\
\frac{d \hat{v}_{c}}{d t}
\end{array}\right]=\left[\begin{array}{cc}
-\frac{\left(r_{L}+D r_{S}+D^{\prime} r_{d}+r_{C}\right)}{L} & -\frac{1}{L} \\
\frac{1}{C} & 0
\end{array}\right]\left[\begin{array}{c}
\hat{l}_{L} \\
\hat{v}_{c}
\end{array}\right]+\left[\begin{array}{ccc}
\frac{D}{L} & \frac{r_{c}}{L} & \frac{V_{i}+\left(r_{d}-r_{S}\right) I_{L}}{L} \\
0 & -\frac{1}{C} & 0
\end{array}\right]\left[\begin{array}{c}
\hat{v}_{i} \\
\hat{\imath}_{o} \\
\hat{d}
\end{array}\right]} \\
& {\left[\begin{array}{c}
\hat{l}_{i} \\
\hat{v}_{o}
\end{array}\right]=\left[\begin{array}{cc}
D & 0 \\
0 & 1+r_{C} C \frac{d}{d t}
\end{array}\right]\left[\begin{array}{c}
\hat{l}_{L} \\
\hat{v}_{c}
\end{array}\right]+\left[\begin{array}{ccc}
0 & 0 & I_{L} \\
0 & 0 & 0
\end{array}\right]\left[\begin{array}{c}
\hat{v}_{i} \\
\hat{l}_{o} \\
\hat{d}
\end{array}\right]}
\end{aligned}
$$

To obtain transfer functions for four g-parameters, the linearized system in (4) is transformed to frequency domain. The open loop analytical expressions in terms of circuit elements are given in the following matrix.

$$
\begin{aligned}
& {\left[\begin{array}{cr}
Y_{i-o} & H_{i-o} \\
G_{o-o} & -Z_{o-o}
\end{array}\right]=\left[\begin{array}{cc}
\hat{i}_{i(s)} & \frac{\hat{i}_{i(s)}}{\hat{v}_{i}(s)} \\
\hat{\imath}_{o}(s) \\
\frac{\hat{v}_{o}(s)}{\hat{v}_{i}(s)} & \frac{\hat{v}_{o}(s)}{\hat{i}_{o}(s)}
\end{array}\right]=\frac{1}{\Delta}\left[\begin{array}{cc}
\frac{D^{2}}{L} & \frac{D\left(1+s r_{C} C\right)}{L C} \\
\frac{D\left(1+s r_{C} C\right)}{L C} & -\frac{\left(1+s r_{C} C\right)\left(r_{L}+D r_{s}+D^{\prime} r_{d}+s L\right)}{L C}
\end{array}\right]} \\
& {\left[\begin{array}{l}
G_{c i} \\
G_{c o}
\end{array}\right]=\left[\begin{array}{c}
\frac{\hat{i}_{i(s)}}{\hat{a}(s)} \\
\frac{\hat{v}_{o}(s)}{\hat{a}(s)}
\end{array}\right]=\frac{1}{\Delta}\left[\begin{array}{c}
s \frac{D\left(V_{i}+\left(r_{d}-r_{s}\right) I_{o}\right)}{L} \\
\frac{\left(1+s r_{c} C\right)\left(V_{i}+\left(r_{d}-r_{s}\right) I_{o}\right)}{L C}
\end{array}\right]+\left[\begin{array}{c}
I_{L} \\
0
\end{array}\right]}
\end{aligned}
$$

where

$$
\Delta=s^{2}+s \frac{r_{L}+D r_{S}+D^{\prime} r_{d}+r_{C}}{L}+\frac{1}{L C}
$$

This is the final mathematical form of derived g-parameter set, whose general representation is given in 
(1). It includes the input admittance, back current gain, audiosusceptibility and output impedance. Table 1 shows the values of different parameters used in the design of buck converter.

Table 1. Buck Converter Parameters

\begin{tabular}{lcc}
\hline \hline Parameters & Symbols & Value \\
\hline Input voltage & $V_{i}$ & $30 \mathrm{~V}$ \\
Output voltage & $V_{o}$ & $10 \mathrm{~V}$ \\
Output current & $I_{o}$ & $1 \mathrm{~A}$ \\
Switching frequency & $f_{S}$ & $100 \mathrm{kHz}$ \\
Capacitor & $C$ & $300 \mathrm{uF}$ \\
Inductor & $L$ & $100 \mathrm{uH}$ \\
\hline \hline
\end{tabular}

\section{Frequency Response Measurement Setup}

Frequency response measurements are very significant for switch mode power supplies. There are certain shortcomings associated with the simulation as it is sometimes unable to show the exact behavior of a power converter. Various effects such as electromagnetic induction (EMI), parasitic of components and noise introduced by different sources cannot be accurately included into the simulation model. Subsequently, it can be said that frequency response measurements are key to the design of switched mode converters [10].

In practice, the network analyzer used for frequency response measurements has operating range from as low as $10 \mathrm{~Hz}$ to several MHz. To measure the frequency response of power supply, certain signals are required to be perturbed. The source of the network analyzer generates sinusoidal perturbation signal to be injected in to the circuit through an isolation transformer. The signal generated from the source of the network analyzer is amplified and then injected in to the circuit, to perturb the input voltage or output current for frequency response measurements. The amplitude of the injection signal should neither be too small so that it is dominated by the noise, nor too large to affect normal operation of the circuit.

Agilent's network analyzer E5061B is used in this work to measure the frequency response of the system [11]. The measurement result plots the gain and phase as a function of frequency. It is important to mention that measurements made by network analyzer are valid up to half of the switching frequency. Measurements made after half the switching frequency are not reliable as the aliasing phenomenon becomes an issue [12].

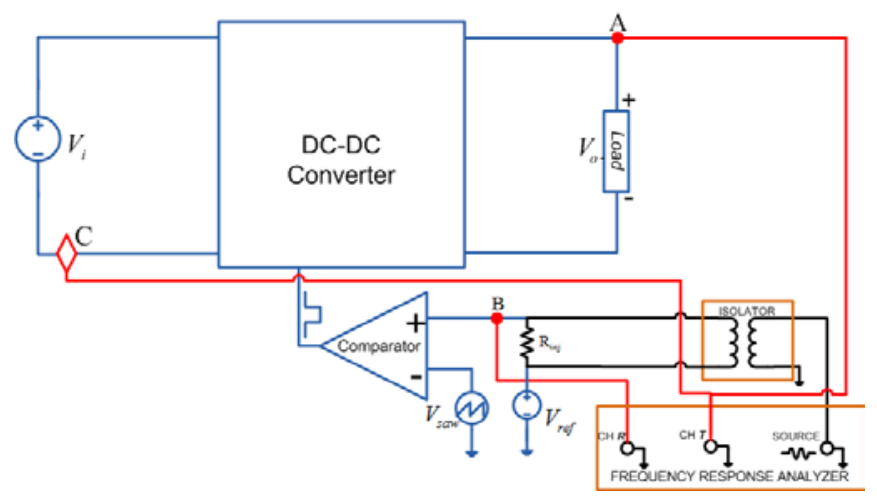

Fig. 3. Measurement setup I for control to output voltage and control to input current.

In order to obtain the control to output voltage transfer function, perturbation in the duty cycle is introduced by adding the injection signal to the reference voltage. The injection resistor is connected in 
series with the dc reference voltage, and applied at the input of comparator. Fig. 3 shows measurement setup I which is used for the frequency response measurement of control to output voltage transfer function and control to input current transfer function [13]. For both these measurements channel $R$ measures the reference voltage. For control to output voltage measurement channel $T$ measures output voltage so the ratio $(A / B)$ gives control to output voltage transfer function $\left(\hat{v}_{o} / \hat{d}\right)$. For control to input current measurement, channel $T$ measures the input current so the ratio $(C / B)$ gives control to input current transfer function $\left(\hat{\imath}_{i} / \hat{d}\right)$.

The measurement setup II shown in Fig. 4, is used for the measurement of output impedance and back current gain [10]. For introducing the perturbation in the output current, the injection signal is connected in parallel. For both these measurements, channel $R$ measures the output current. For output impedance measurement, channel $T$ measures output voltage so the ratio $(A / B)$ gives output impedance $\left(\hat{v}_{o} / \hat{l}_{o}\right)$. For back current gain measurement channel $T$ measures the input current so the ratio $(C / B)$ gives back current gain $\left(\hat{l}_{i} / \hat{l}_{o}\right)$.

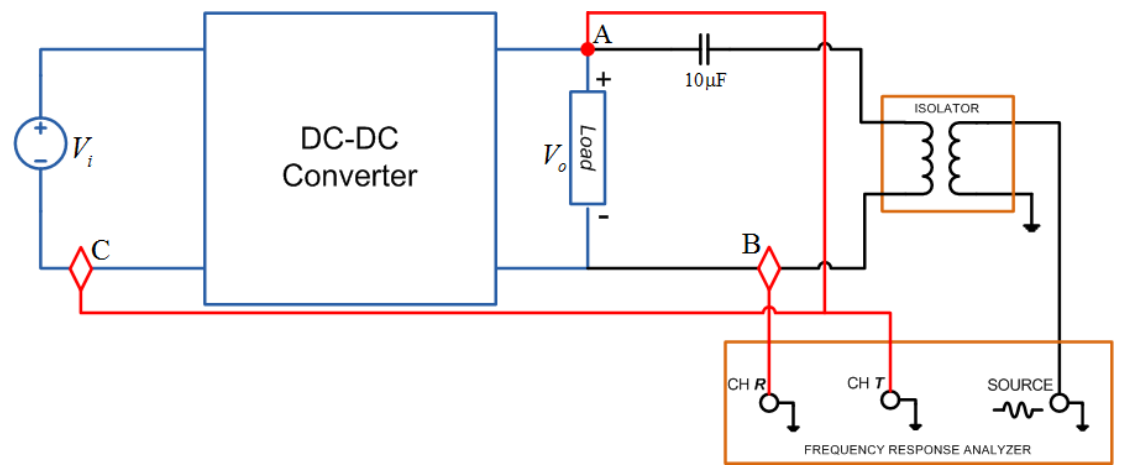

Fig. 4. Measurement setup II for output impedance and back current gain.

The measurement setup III shown in Fig. 5, is used for the measurement of input admittance and audiosusceptibility [10]. Compared to the output impedance and back current gain measurements, it is more difficult to measure the audiosusceptibility and input admittance because the perturbation is to be added to input voltage. For introducing perturbation in the input voltage, the injection signal is connected in series with the source. For both these measurements, channel $R$ measures the input voltage. For input admittance measurement, channel $T$ measures the input current so the ratio $(C / B)$ gives input admittance $\left(\hat{\imath}_{i} / \hat{v}_{i}\right)$. For audiosusceptibility measurement channel $T$ measures the output voltage so the ratio $(A / B)$ gives audiosusceptibility $\left(\hat{v}_{o} / \hat{v}_{i}\right)$.

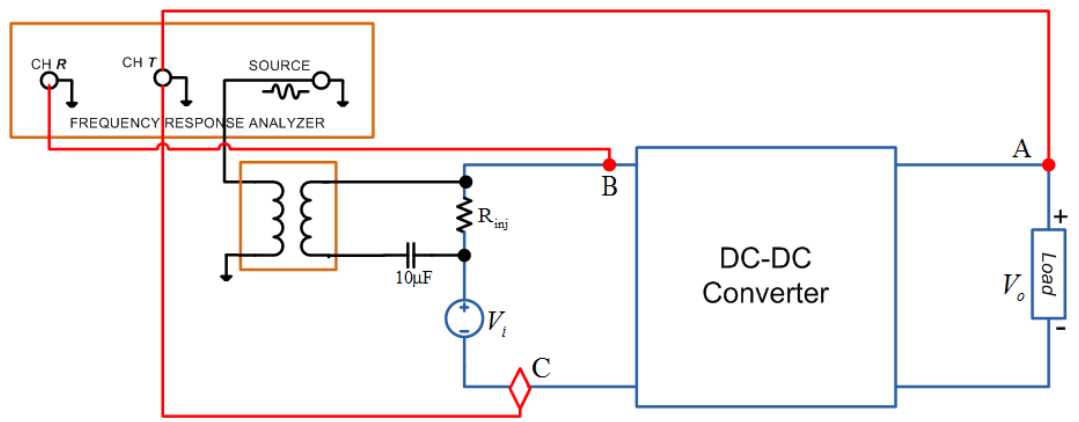

Fig. 5. Measurement setup III for input admittance and audiosusceptibility.

\section{Frequency Response Measurement Results}

The control to output voltage transfer function is a measure of the sensitivity of output voltage to the variations in the duty cycle, while the input voltage and load current are kept constant. The control to input 
current frequency response determines how the input current is related to duty cycle. Fig. 6 shows the frequency response for both these transfer functions obtained using measurement setup I, compared with the analytical expressions with and without the effect of parasitic elements.

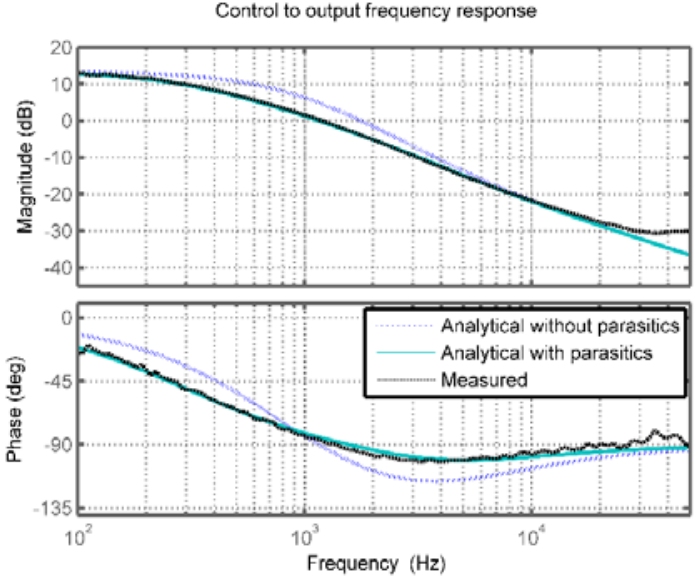

(a)

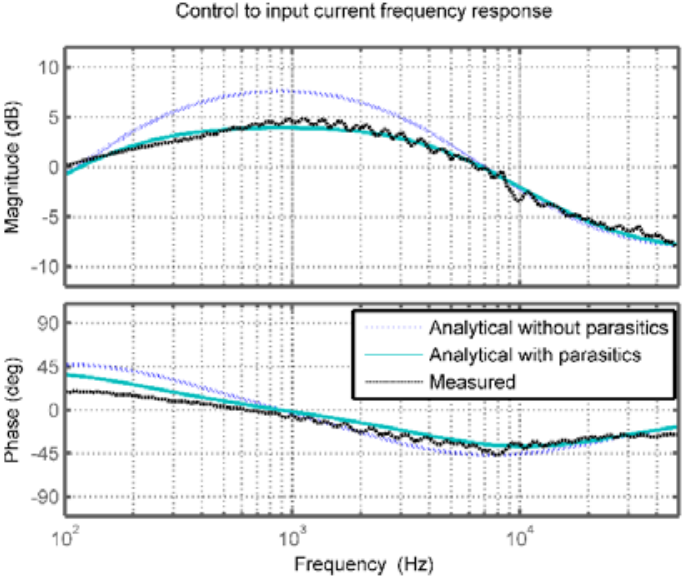

(b)

Fig. 6. Frequency response comparison of (a) Control to output voltage (b) Control to input current.

\subsection{Frequency Response Measurement of g-Parameters}

There are four important transfer functions that are used for the complete characterization of a switch mode power supply. The results from measurement as well as analytical expressions for each of them in open loop as well as closed loop are given in the following section.

The output impedance frequency response measurement contains information regarding response of the converter to dynamic load changes at different frequencies. Fig. 7 shows the frequency response for open loop output impedance and back current gain obtained using measurement setup II.

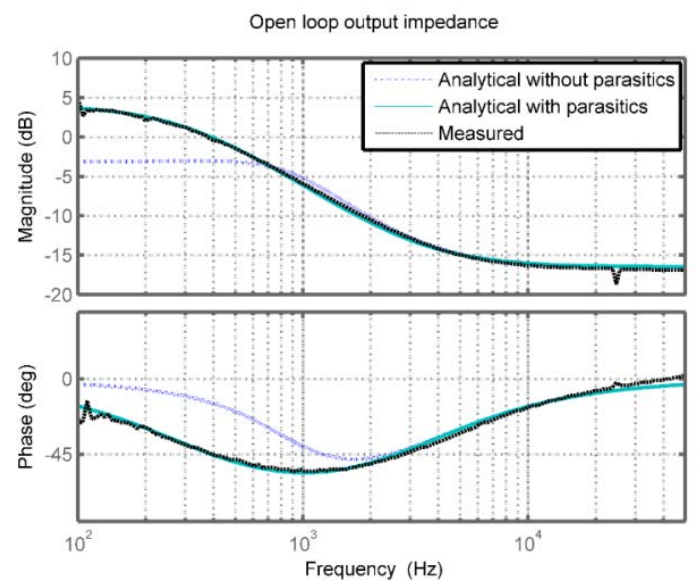

(a)

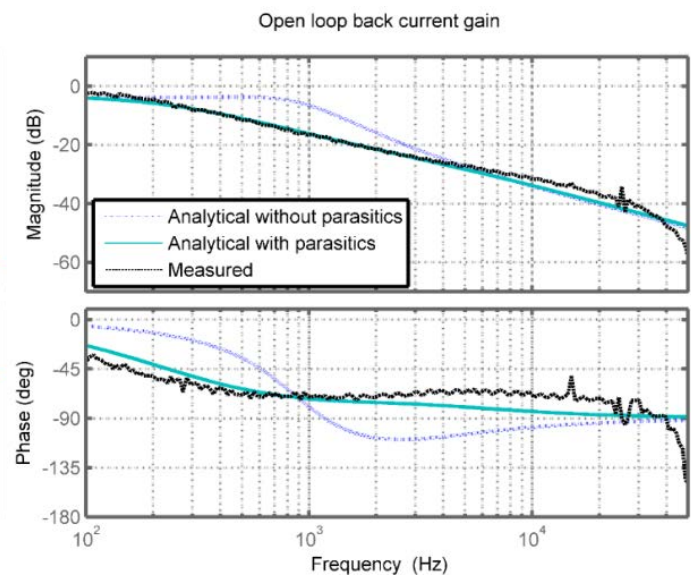

(b)

Fig. 7. Frequency response comparison of open loop (a) Output impedance (b) Back current gain.

Audiosusceptibility frequency response measurement determines the transmission of noise from the input of the system to the output. It tells about the ability of the converter to reject noise appearing at the input.

As the output impedance gives an idea about any changes in load, input impedance does so about any interaction from the source. It determines the sensitivity of power system to input filter or input power components. Fig. 8 shows the frequency response for open loop audiosusceptibility and input admittance 
obtained using measurement setup III.

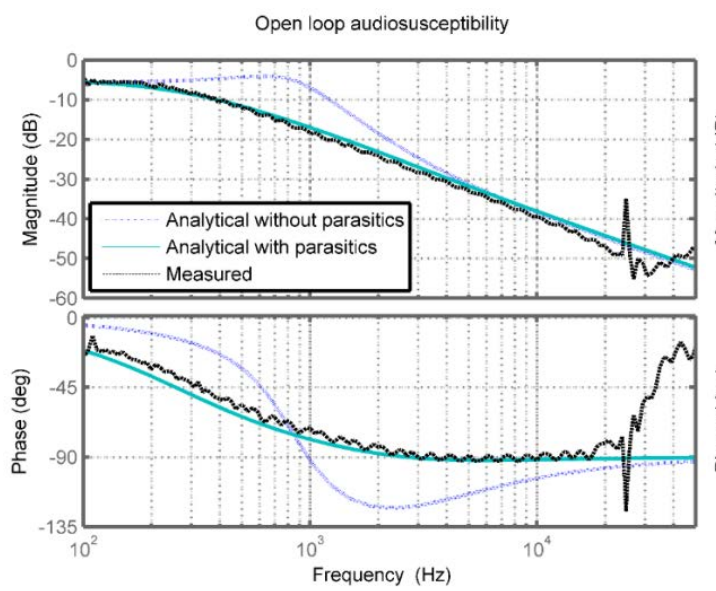

(a)

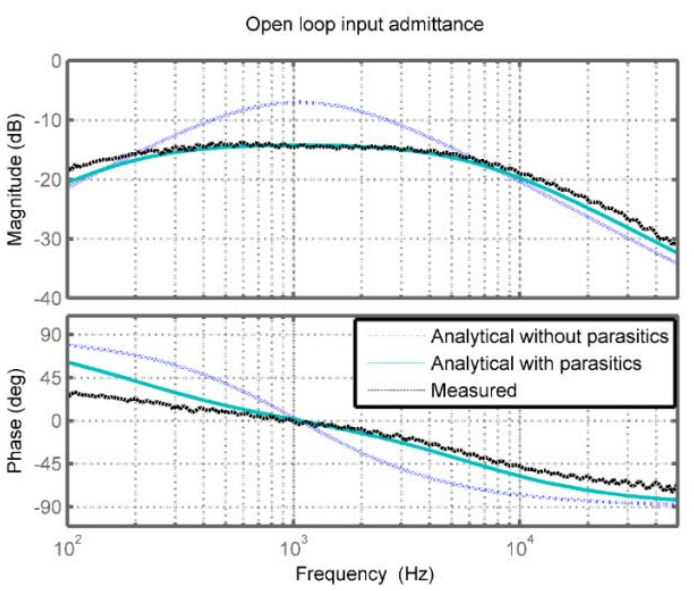

(b)

Fig. 8. Frequency response comparison of open loop (a) Audiosusceptibility (b) Input admittance.

After having compared the open loop measurement results with corresponding analytical expressions, the comparison of results for closed loop system is shown in Fig. 9 for four g-parameters.

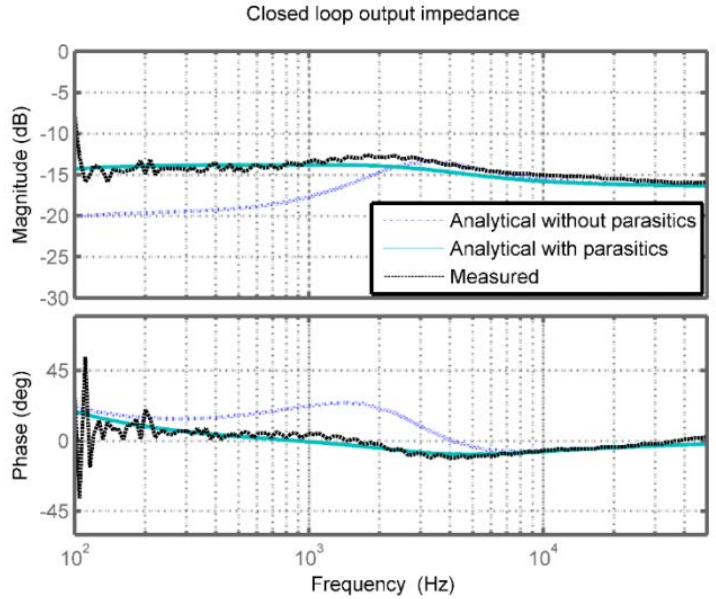

(a)
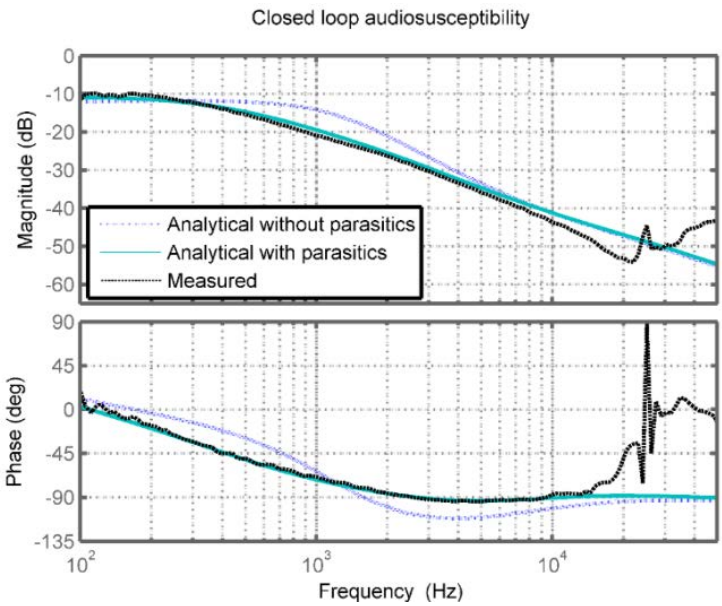

(c)

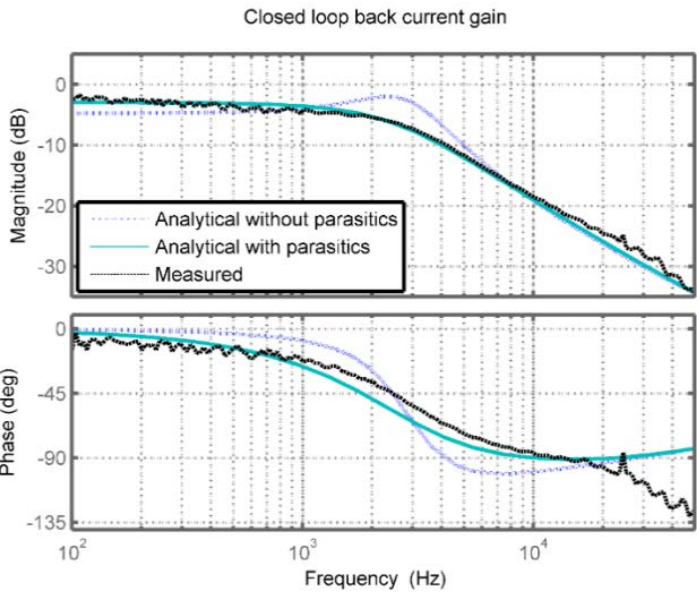

(b)

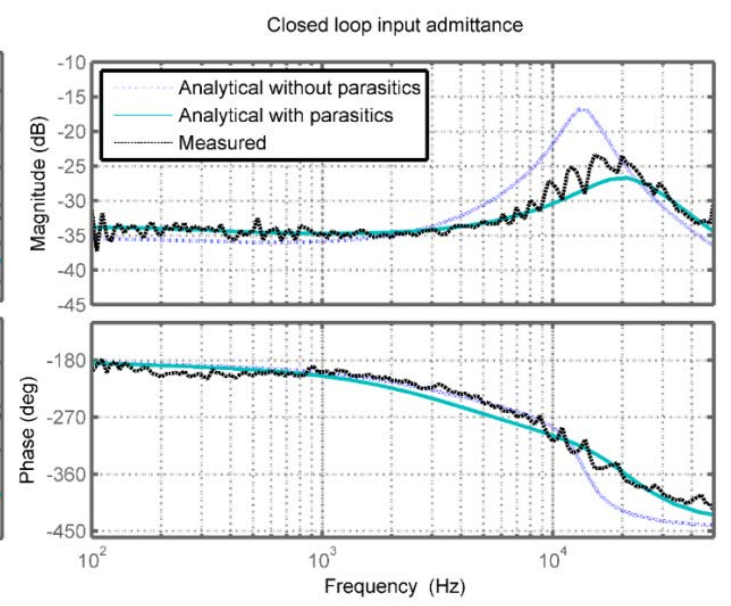

(d)

Fig. 9. Frequency response comparison of closed loop (a) Output Impedance (b) Back current gain (c) Audiosusceptibility (d) Input admittance. 


\subsection{Frequency Response of Filter Components}

The frequency response measurement and calculation of the parasitic resistance for the output filter components i.e. capacitor and inductor is explained in this section [10].

The objective of measuring frequency response for a capacitor is to determine the exact value of its equivalent series resistance (ESR). It is necessary to have precise idea about the ESR of the capacitor as it is also used in the controller design process. The setup used for the frequency response measurement of capacitor is shown in Fig. 10. The solid line represents the measurement probe while the dotted line represents reference or ground probe.

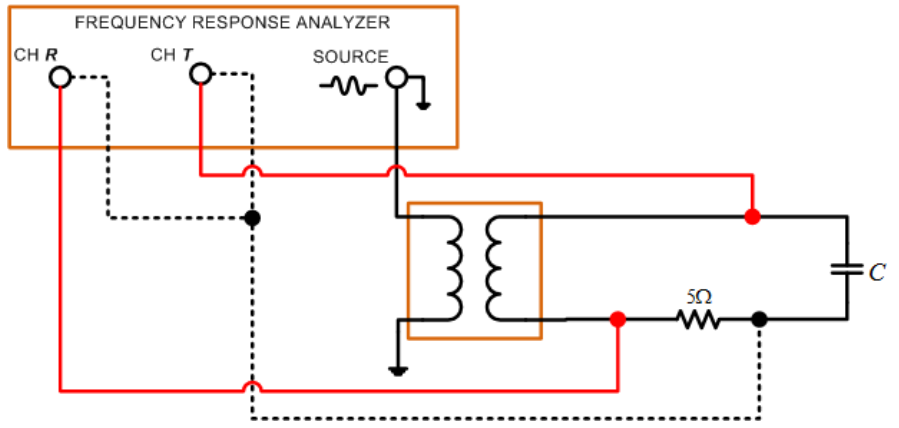

Fig. 10. Measurement setup for frequency response of capacitor.

The transfer function for capacitor without any ESR can be written as

$$
X_{c}=\frac{1}{S_{c}}
$$

The addition of ESR modifies the transfer function to

$$
X_{r c}=r_{c}+\frac{1}{s_{c}}
$$

It can be written in pole zero form as

$$
X_{r c}=r_{c}\left(\frac{s+\frac{1}{r_{c} C}}{s}\right)
$$

By equating the numerator to zero we can find the location of zero frequency $\left(f_{r c}\right)$ caused by ESR as given below

$$
f_{r c}=\frac{1}{2 \pi r_{c} C}
$$

Conversely we can find the value of ESR by

$$
r_{c}=\frac{1}{2 \pi f_{r c} C}
$$

Now, if we get the frequency response plot for capacitor with certain ESR value, it is possible to determine the exact value of capacitor's ESR using this plot.

The comparison plot in Fig. 11 for a capacitor with and without ESR quite clearly highlights the difference in the frequency response caused by the addition of an extra zero to the transfer function by the ESR of the capacitor. The capacitor without ESR has a classical single pole frequency response with $-20 \mathrm{~dB} / \mathrm{dec}$ roll off in magnitude response and constant value of $-90^{\circ}$ in the phase plot. The capacitor with a certain value of ESR follows the plot without ESR till the effect of ESR starts taking over. The ESR causes an extra zero to be 
added to the system. Generally a single zero causes the magnitude frequency response to rise with $+20 \mathrm{~dB} / \mathrm{dec}$ and phase frequency response to have phase $+90^{\circ}$. So the addition of zero here cancels the effect of pole and makes the magnitude response plot to have roll off of $0 \mathrm{~dB}$ and in the phase plot, phase changes to $0^{\circ}$. The exact location of the frequency of this zero $\left(f_{r c}\right)$ caused by ESR can be easily determined from the phase plot. It is the value at which phase in $-45^{\circ}$. Then we can determine the value of ESR using (10).

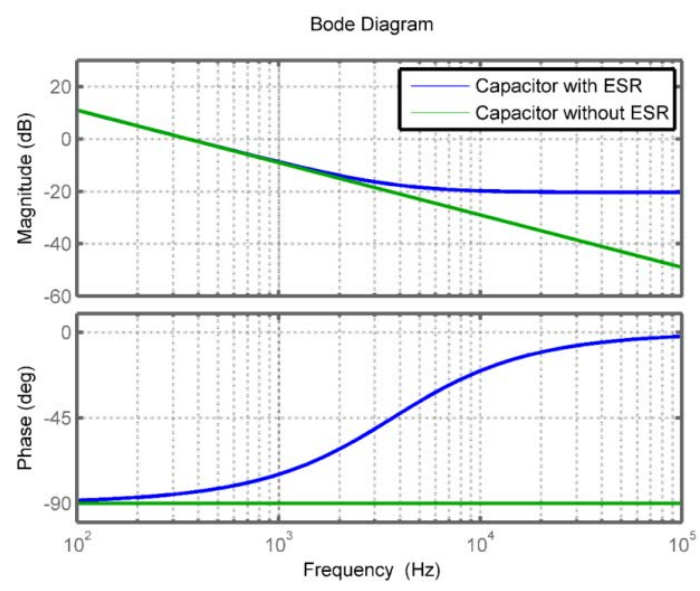

Fig. 11. Frequency response comparison for capacitor with and without ESR.

The procedure illustrated above is validated with the practical measurement of frequency response of a capacitor. We take a capacitor $(C=450 u F)$ with an unknown value of ESR. The frequency response measurement of the capacitor is done using the measurement setup given in Fig. 10.

As explained above, first we look at the phase plot and determine the frequency at which phase has a value of $-45^{\circ}$ (or $\left.135^{\circ}\right)$. This is the frequency at which zero is located $\left(f_{r c}\right)$. Once we get the value of $\left(f_{r c}\right)$ we can easily determine the value of $\left(r_{c}\right)$ using (10).

$$
r_{c}=\frac{1}{2 \pi f_{r c} C}=\frac{1}{2 \pi(3.68 k)(450 u)}=96 \mathrm{~m} \Omega
$$

After having obtained the value of ESR we plot the result from the measurement together with the simulation result of capacitor with $\left(C=450 u F\right.$ and $\left.r_{c}=96 \mathrm{~m} \Omega\right)$ using (7). The close agreement of results in Fig. 12 validates the procedure followed for ESR calculation.

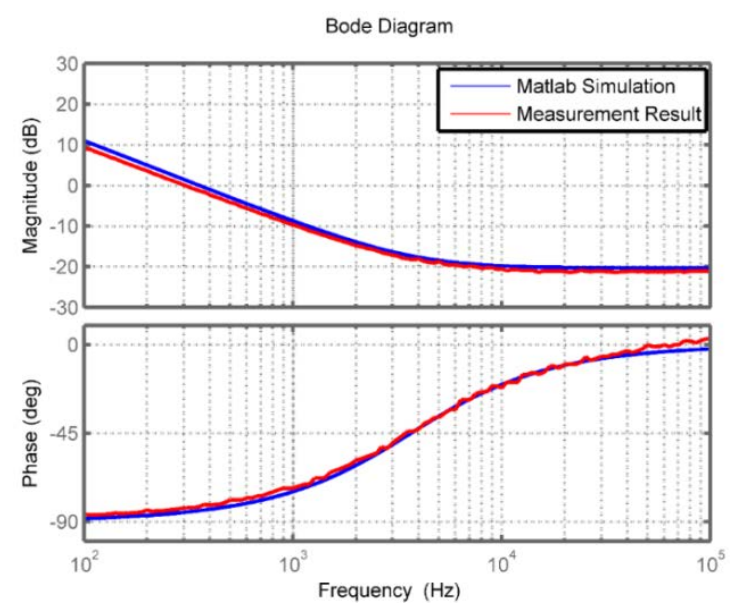

Fig. 12. Frequency response of capacitor. 


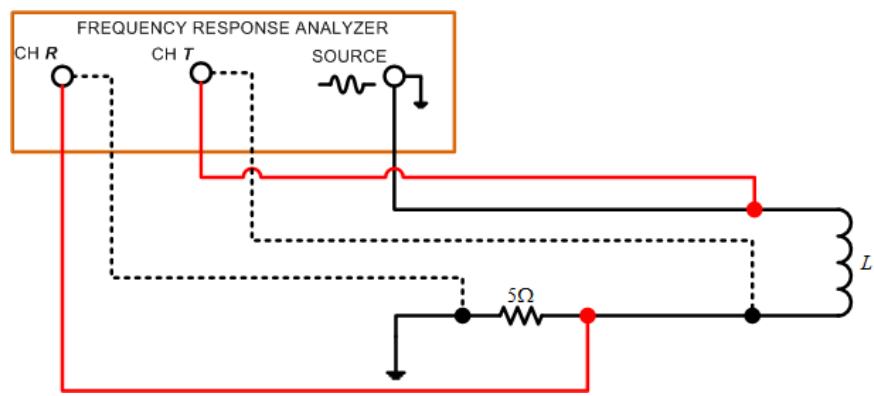

Fig. 13. Measurement setup for frequency response of inductor.

The measurement of frequency response for an inductor helps us to determine the value of parasitic resistance associated with the inductor. The setup used for the frequency response measurement of inductor is shown in Fig. 13. The solid line represents measurement probe while the dotted line represents reference or ground probes.

The transfer function for inductor without any DCR can be written as

$$
X_{L}=s L
$$

The addition of parasitic resistance modifies the transfer function to

$$
X_{r L}=r_{L}+s L
$$

Unlike the capacitor, where its parasitic resistance adds another zero to the transfer function, the parasitic resistance of inductor just shifts the position of frequency of zero from origin to negative real axis. From (12) we can find the location of zero frequency $f_{r L}$

$$
f_{r L}=\frac{r_{L}}{2 \pi L}
$$

Conversely we can find the value of parasitic resistance by

$$
r_{L}=2 \pi f_{r L} L
$$

Now, if we get the frequency response plot for inductor with certain parasitic resistance value, it is possible to determine the exact value of inductor's parasitic resistance from this plot.

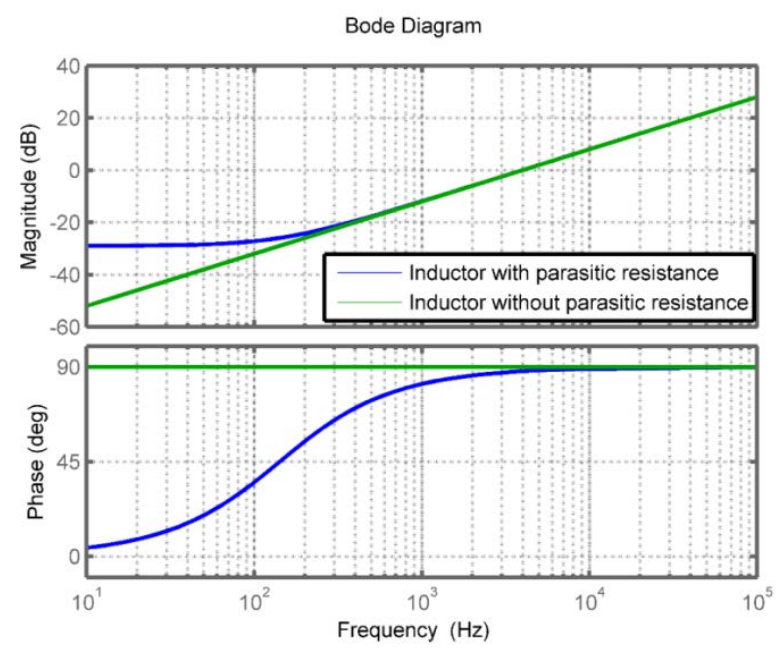

Fig. 14. Frequency response comparison for inductor with and without DCR.

The comparison plot in Fig. 14 for an inductor with and without parasitic resistance clearly shows the shift in the position of zero caused by series resistance of inductor. The change in the value of the frequency 
of zero $f_{r L}$ caused by parasitic resistance can be easily determined from the phase plot. It is the value at which phase is $+45^{\circ}$. Then we can determine the value of parasitic resistance using (14).

The procedure illustrated above is validated through measurement of frequency response and through the calculation of parasitic resistance for an inductor. We take an inductor $(L=40 u H)$ with an unknown value of parasitic resistance. The frequency response measurement of the inductor is done using the measurement setup given in Fig. 13.

As explained above first we look at the phase plot and determine the frequency at which phase has a value of $+45^{\circ}$. This is the frequency $f_{r L}$ at which zero is located.

Once we get the value of $f_{r L}$ we can easily determine the value of $r_{L}$ using (14).

$$
r_{L}=2 \pi(142)(40 u)=35.7 m \Omega
$$

After having obtained the value of parasitic resistance we plot the result from measurement together with the simulation result of inductor using (12) in Fig. 15 with $\left(L=40 u H\right.$ and $\left.r_{L}=35.7 \mathrm{~m} \Omega\right)$. The close agreement of results validates the procedure followed for calculation parasitic resistance associated with inductor.

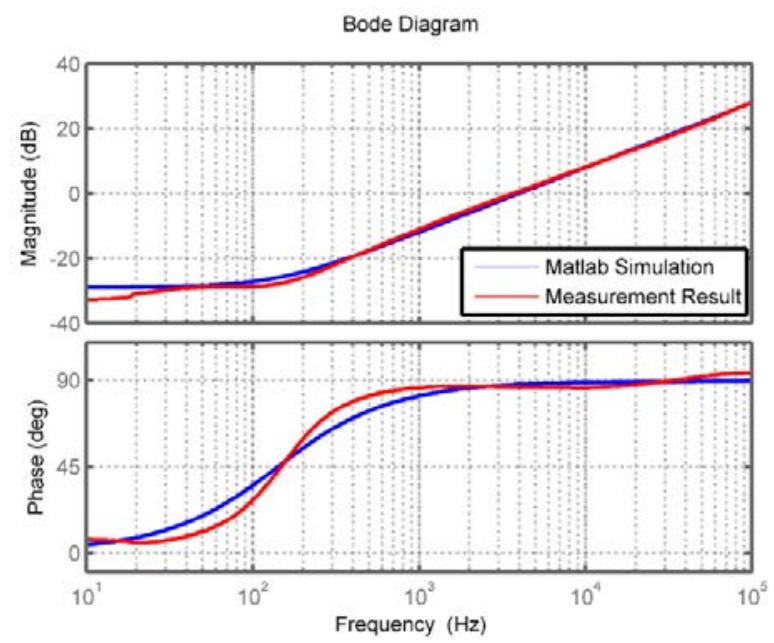

Fig. 15. Frequency response of inductor.

\section{Conclusion}

Small signal models are developed for the analysis of dc-dc converters, which are integral part of modern distributed power systems. High accuracy is desired for the correct analysis of complete system using these models. The inclusion of parasitic elements makes the modeling process complex but increases the accuracy of models. A simple procedure is illustrated and experimentally validated for the measurement of series resistance associated with capacitor and inductor. The small signal model is experimentally validated by the measurement of different frequency responses for open and closed loop system of prototype dc-dc buck converter. The frequency response results from the measurement are compared with those obtained using analytical expressions and it is found that the results match with analytical results very well. Hence this procedure can be used for the validation of the modeling procedure of the converter.

\section{References}

[1] Ortiz, C. E. C. (2005, Sep.). Circuit oriented average modeling of switching power converters. 
Proceedings of European Conference on Power Electronics and Applications (pp. 11-14).

[2] Abramovitz, A. (2011, Aug.). An approach to average modeling and simulation of switch-mode systems. IEEE Trans. Educ., 54(3), 509-517.

[3] Izquierdo, D., Azcona, R., Cerro, F. J. D., Fernandez, C., \& Delicado, B. (2010, Feb.). Electrical power distribution system (HV270DC), for application in more electric aircraft. Proceedings of 25th Annual IEEE Applied Power Electronics Conference and Exposition (pp. 1300-1305).

[4] Boroyevich, D., Cvetkovic, I., Dong, D., Burgos, R., Wang, F., \& Lee, F. (2010). Future electronic power distribution systems a contemplative view. Proceedings of 12th International Conference on Optimization of Electrical and Electronic Equipment (OPTIM) (pp. 1369-1380).

[5] Siegers, F., Arrua, S., \& Santi, E. (2015, Sep.). Stabilizing controller design for multi-bus MVDC distribution systems using a passivity based stability criterion and positive feed-forward control. Proceedings of IEEE Energy Conversion Congress and Exposition (ECCE) (pp. 5180-5187).

[6] Suntio, T. (2009). Dynamical Profile of Switched Mode Converter; Modeling, Analysis and Control (1st ed.). Wiley.

[7] Suntio, T. (2006, Feb.). Unified average and small signal modeling of direct-on-time control. IEEE Transactions on Industrial Electronics, 53(1), 287-295.

[8] Amedo, L., Boroyevich, D., Burgos, R., \& Wang, F. (2008). Un-terminated frequency response measurements and model order reduction for black box terminal characterization models. Proceedings of Twenty-Third Annual IEEE Applied Power Electronics Con. and Exposition (APEC) (pp. 1054-1060).

[9] Hankaniemi, M., \& Suntio, T. (2006). Small signal models for constant-current regulated converters. Proceedings of IEEE 32nd Annual Conference on Industrial Electronics (IECON) (pp. 2037-2042).

[10] Ridley, R. (2006). Measuring frequency response: Tips and methods. Design Center Library, Switching Power Magazine. Retrieved from the website: http://www.ridleyengineering.com

[11] Agilent Technologies. Keysight electronic measurement products. Retrieved from the website: http://www.agilent.com

[12] Verghese, G. C., \& Thottuvelil, V. J. (1999). Aliasing effects in PWM power converters. Proceedings of IEEE Power Electronics Specialists Conference (pp. 1043-1049).

[13] Swaminathan, B., \& Ramanarayanan, V. (2006, Aug.). Application of network analyzer in measuring the performance functions of power supply. Journal of Indian Institute of Science, 86, 315-332.

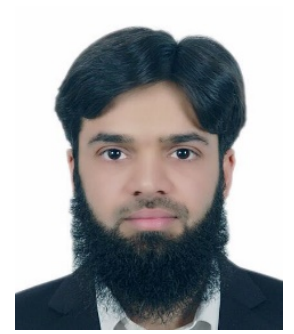

Husan Ali received his B.Sc. degree in electrical engineering and his M.Sc. degree in communication and electronics engineering from the University of Engineering and Technology (UET), Peshawar, Pakistan in 2009 and 2012 respectively. He received his M.Sc. degree in electrical engineering from Northwestern Polytechnical University (NPU), Xi'an, China in 2014, where he is presently working towards his Ph.D. degree in the School of Automation. His current research interests include behavioral modeling and analysis of distributed power systems.

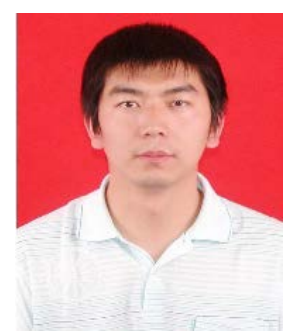

Xiancheng Zheng received his B.Sc., M.Sc. and Ph.D. degrees in electrical engineering from Northwestern Polytechnical University (NPU), Xi'an, China in 1998, 2001 and 2011 respectively. In 2001, he joined NPU, where he is presently working as a professor in the School of Automation. His current research interests include bidirectional AC-DC converter designs for aircraft electrical power systems, and silicon carbide based high power density DC-DC and DC-AC converters. 


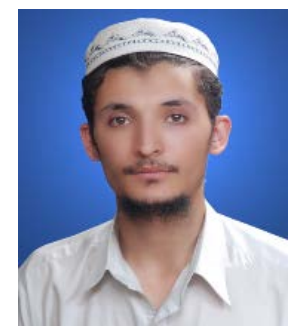

Shahbaz Khan received his B.Sc. degree in electronics engineering from the University of Engineering and Technology (UET), Abbottabad, Pakistan in 2010; received his M.Sc. degree in electrical engineering from Northwestern Polytechnical University (NPU), Xi'an, China in 2014, where he is presently working towards his Ph.D. degree in the School of Automation. His current research interests include the modeling of AC-DC-AC converters and their stability analysis.

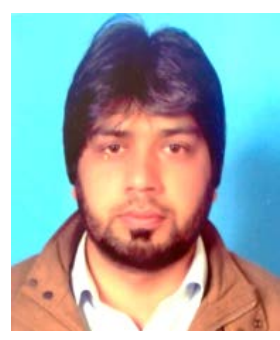

Haider Zaman received his B.Sc. degree in electronics engineering from the University of Engineering and Technology (UET), Abbottabad, Pakistan in 2008; received his M.Sc. degree in electrical engineering from the COMSATS Institute of Information Technology (CIIT), Abbottabad, Pakistan in 2013. He is presently working towards his Ph.D. degree in the School of Automation, Northwestern Polytechnical University, Xi'an, China. His current research interests include silicon carbide based high power density DC-DC and DC-AC converters.

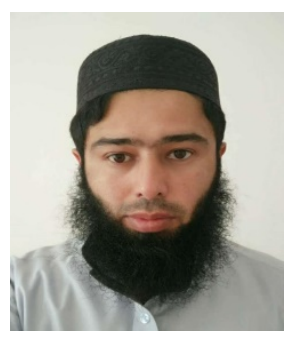

Muhammad Saad received his B.Sc. degree in electronics engineering from the University of Engineering and Technology (UET), Abbottabad, Pakistan in 2010; and his M.Sc. degree in electrical engineering from Northwestern Polytechnical University (NPU), Xi'an, China in 2016. He is presently working towards his Ph.D. degree in the School of Electronics and Control Engineering, Changán University, Xi'an, China. His current research interests include the impedance measurement and modeling of three phase systems. 